\title{
Simulation and optimization for ship lock scheduling: a case study
}

\author{
Jannes Verstichel \\ $K U$ Leuven, Department of Computer Science \\ CODeS \& iMinds \\ Gebroeders De Smetstraat 1, 9000 Gent, Belgium \\ Email: jannes.verstichel@cs.kuleuven.be
}

\author{
Greet Vanden Berghe \\ KU Leuven, Department of Computer Science \\ CODeS \& iMinds \\ Gebroeders De Smetstraat 1, 9000 Gent, Belgium \\ Email: greet.vandenberghe@cs.kuleuven.be
}

\begin{abstract}
The influence of infrastructural modifications on port and waterway locks to the lock system's capacity cannot be estimated accurately. This paper introduces a simulation model and optimization approach to ship lock scheduling capable of simulating the effects of infrastructural changes upon traffic handling at a ship lock with parallel chambers. Simulation parameters are determined via an analysis of historical data. The model's is ascertained via a simulation of the current operations at the lock. The effect of infrastructural changes upon traffic handling may then be accurately assessed via new simulations. The downtimes of lock chambers due to canal drainage requirements, for example, represent a particularly interesting element of the simulation's input. Optimization of traffic handling in the new infrastructural environment is consequently performed to increase the ship lock's throughput and decrease passage times for ships. Results demonstrate the huge influence drainage policy has upon passage times, how optimizing the process results in greatly improved lock traffic handling and finally how it is in lock proprietors' best interest to seriously consider alternatives for chamber drainage downtimes.
\end{abstract}

\section{Introduction}

Ship locks represent a crucial element of maritime infrastructure. They separate tide-independent ports from tidedependent rivers, ensuring stable water levels in docks. Locks are also employed on inland waterways to control both the draft and water flow of rivers or canals, allowing large ships to travel far into the hinterland. Ship locks thereby enable the construction of artificial canals navigable with minimal water consumption. Locks are used to transfer ships from one water level to another, and have played a major role in enabling the economic development engendered by worldwide maritime transportation. They also represent a crucial component of inland waterway transportation, currently considered one of the most ecological alternatives for road transportation [1], [2]. With the steady rise in both the size and number of ships passing through locks, however, they increasingly represent a major bottleneck in marine transportation [?], [3]. Existing lock infrastructure is not only quite old, but many locks are also rapidly becoming too small for the ships they must transfer. The rising need for new, larger, faster and more efficient ship locks is forcing lock owners to either renovate existing lock chambers or construct new ones. Thorough lock renovations, however, require long chamber downtimes, negatively influencing traffic handling capabilities during such periods. Building new locks is possible with less (or no) downtime, but, when space is scarce, building a new lock chamber might require the destruction of an existing one. The influence of such long downtimes is currently only roughly estimated and both proprietors and shippers can only hope for the best as regards incurred delays. It is difficult to devise and test policies to reduce passage times in advance, since conducting live tests corresponds to long and unnecessary downtimes. Furthermore, the high-level simulation software currently available is unable to capture enough detail to accurately simulate traffic handling after large changes in lock infrastructure.

This paper presents a detailed simulation model and optimization approach, implemented in a software tool called MOGLi (Multilevel Optimization and Generic models for Lock scheduling). The results of applying MOGLi to determine the effect of different infrastructural changes on traffic handling is discussed for a case study.

\section{MOGLi}

MOGLi incorporates a detailed simulation and optimization tool for ship lock scheduling containing both an exact solver and meta-heuristic libraries. In simulation mode, the software generates solutions that approximate current practice at ship locks, calibrates its parameters using historical data and investigates the effect of changes (in infrastructure, traffic density, traffic composition, ...) on existing traffic handling policies. The simulation generates solutions using constructive heuristics based on the first-come-first-served principle that iteratively assigns each ship to the chamber which generates the smallest passage time for the ship and, if relevant, previously assigned ships for this chamber. In optimization mode, MOGLi employs either exact solvers or meta-heuristics. The exact solvers apply models based on integer programming formulations for the ship placement 
problem [4] and lock scheduling problem [5], and Combinatorial Benders' decomposition for the lock scheduling problem [6], and the results are obtained via standard integer programming solvers such as Gurobi [7] and CPLEX [8]. While these solvers are capable of optimally solving small to medium sized instances, they prove incapable of solving real-life instances for locks with parallel chambers or when the planning horizon becomes large. The meta-heuristic optimization libary can, by contrast, provide high quality (albeit not necessarily optimal) solutions for instances of any size for any lock configuration. Beginning from the simulation solution, meta-heuristics adjust the ship-to-chamber assignments and ship sequence to reduce both maximum and average passage times. The meta-heuristic library contains efficient implementations of Variable Neighbourhood Search [9], Simulated Annealing [10] and Late Acceptance [11], applies the corridor method for efficient neighbourhood exploration [12] and guide and observe [14] with dynamic objective adjustments for intensification and diversification.

\section{Case study}

In this paper MOGLi is applied to a real case where the smallest chamber in a three-chamber ship lock is replaced by a new, larger chamber. During the destruction of the old chamber and construction of its replacement, the already highly utilized lock complex must operate with only its two remaining chambers. Existing high level simulation tools were unable to provide reliable predictions for passage times during these infrastructural changes, therefore MOGLi was applied to provide a more accurate prediction.

All ship passages and lock operations were logged for a period of several months, capturing both low and high traffic periods. This provided enough data to calibrate MOGLi and enable accurate simulation and optimization for both the existing and future lock configuration. The data included ship arrival times, ship dimensions and types, the usage of tow boats and the timing information of lockage operation for each transferred ship, such as the start and end time of the lockage and time required for all ships to sail out from the chamber.

Based on the historical timing data, standard lockage durations for each chamber were determined using the logged lockage duration of empty lockages (turnback operations where no ships are transferred). The sailing time required for ships to enter and exit lock chambers was also determined. These times may vary for each chamber and ship type (sea ship, barge), and are further dependent on the number of ships of each type simultaneously transferred. The sailing time required for the fourth barge to enter the chamber may, for example, differ from the sailing time required for the fifth barge to enter. It should be noted that not all data was logged automatically, and that a certain percentage of the data proved invalid. This becomes clear from the example in Figure 1, which illustrates the time required for all ships to exit the chamber after the lockage operation. Despite transferring up to 13 ships in a single lockage operation, the majority of records claim the time required to let all

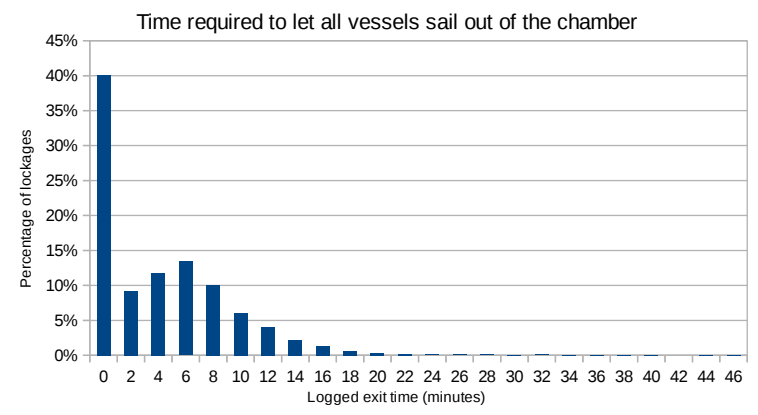

Figure 1. Distribution of logged exit times for one of the lock's chambers. The large peak at zero minutes is a result of errors in the semi-manual data logging.

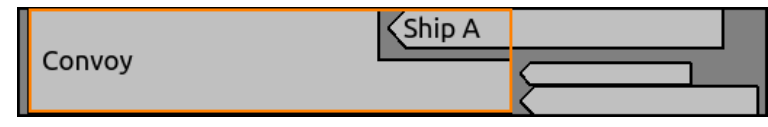

Figure 2. Example of a convoy with some free space, and how this space may be exploited to add more ships to the lockage.

ships sail from the chamber is zero minutes. To increase data analysis accuracy, all records containing such physically impossible or clearly invalid values were ignored.

Based on the data concerning each ship's lockage, it was possible to determine which ships had been transferred together in a single lockage operation. This enabled the callibration of mooring rules (which ship types are permitted to moor to each other) and safety distances (the distance required between ships inside the chamber to ensure a safe lockage operation) for each chamber. Mooring and safety distance rules were assigned values which enabled the simulation to reproduce $>98 \%$ of the historical lockages. The simulation was, however, frequently unable to reproduce lockages that transferred one or more convoys $(\sim 77 \%)$. This is due to how convoys (a collection of motorized and unmotorized barges tied together into a single vessel) often have some free space in their configuration, as shown in Figure 2. While lock operators regularly exploit this free space to increase the chamber's filling rate, the system logs the convoy's entire rectangular dimension (shown in orange within Figure 2). Therefore, while manual operators intuitively override the logged dimensions, MOGLi cannot exploit a convoy's true configuration and dimensions to maximize the filling rate and reproduce the lockage. For example, Ship A within Figure 2 would not be permitted to join the lockage in the simulation despite the ability for it to fit in practice.

The historical data also contained information concerning downtimes of the chambers due to faults, maintenance, and most importantly, drainage. Lock proprietors must drain when the water level on the upstream side of the lock is too high. When this occurs, both doors of a single lock chamber are opened and the excess water flows to the downstream side of the lock. During this drainage operation the lock chamber is unavailable for ship transfer, and thus drainage 
has a significant influence on the traffic handling, especially when periods of heavy rainfall coincide with heavy traffic. One interesting aspect of these drainage downtimes is that they can be avoided by installing a dedicated drainage system in the lock. This drainage system removes the need to drain via the lock chambers, but requires the instalment of large sewage pipes and control systems. When the expected increase in passage times at the lock due to drainage downtimes is sufficiently large, it may prove sensible for lock proprietors to install such a drainage system despite the additional cost, as it would greatly improve the quality of service at the lock. This paper consequently focuses on the influence of drainage upon traffic handling.

\section{Simulation}

The simulation's first aim is calibrating MOGLi such that it generates realistic solutions for the lock's current setup of three parallel chambers. This aim was achieved by setting all parameters using the results of the historical data analysis results before fine-tuning both the locks' and simulation's constructive heuristic's parameters. This simulation was conducted for both a situation where all chambers have an uptime of $100 \%$ (no downtimes due to drainage, faults or maintenance) and where the logged downtimes were included in the simulation. The simulation's second goal was determining the effect of infrastructural changes on passage times under an unchanged traffic handling policy.

In the remainder of this paper, the original setup with three parallel chambers will be referred to as the ' $3 \mathrm{C}$ setup'. The new setup with only two parallel chambers will be referred to as the ' $2 \mathrm{C}$ setup'. Any setup with a dedicated drainage system is referred to as ' $100 \%$ uptime', while setups where the lock proprietors drain via the lock chambers is referred to as 'drainage downtimes'.

\subsection{C Setup}

Passage times from the different simulations for the 3C setup are included in Table 1. All times are expressed relative to the logged times from the historical data, which are employed as the reference point of $100 \%$. Evidently, ignoring downtimes results in a serious underestimation of maximum lock passage times. Including only the downtimes due to drainage results in smaller underestimations. When considering all chamber downtimes, the simulation's results are very close to the historical ones, both with respect to the maximum, average and median passage times. This becomes further evident from Figure 3 which plots the distribution of passage times for the historical data (blue) and compares it against the distribution obtained from the simulation (red). Except for differences between historical and simulation results regarding the number of ships with passage times between 20 and 30 minutes and between 40 and 60 minutes, both curves are very similar, highlighting the accuracy of the simulation.
TABLE 1. PASSAGE TIMES FOR DIFFERENT 3C SIMULATIONS, RELATIVE TO THE LOGGED PASSAGE TIMES FROM HISTORICAL DATA $(100 \%)$.

\begin{tabular}{l|ccc} 
Passage time & $100 \%$ uptime & drainage downtimes & all downtimes \\
\hline Maximum & $64 \%$ & $69 \%$ & $106 \%$ \\
Average & $94 \%$ & $97 \%$ & $101 \%$ \\
Median & $96 \%$ & $100 \%$ & $104 \%$
\end{tabular}

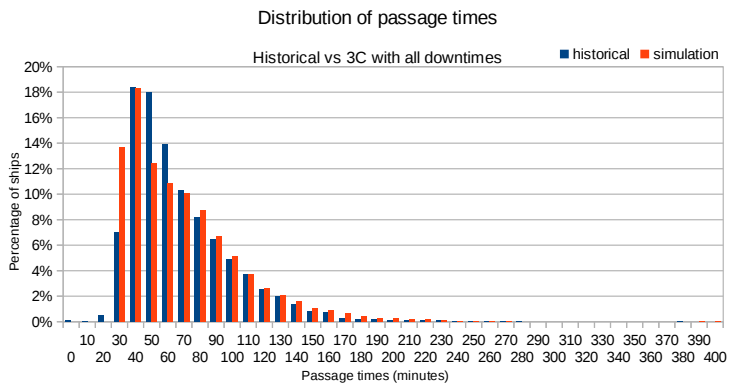

Figure 3. Distribution of passage times at the lock for both the historical data (blue) and the $3 \mathrm{C}$ simulation with all downtimes (red).

\subsection{C Setup}

Passage times from the different $2 \mathrm{C}$ setup simulations where the smallest chamber is permanently unavailable, are provided by Table 2 . All times are expressed relative to the logged times from the historical data, which are used as the reference point of $100 \%$. The removal of the smallest chamber roughly doubles the maximum and average passage times at the lock when the two remaining chambers maintain $100 \%$ uptime (i.e. when a dedicated drainage system is installed). This indicates that, despite the removed chamber being the smallest of the three, it makes a very significant difference regarding overall lock capacity. This is confirmed by Figure 4 which plots the distribution of passage times for the historical data (blue) and compares it against the distribution obtained from the simulation (red). The passage time distribution continues to exhibit a peak at around 30 minutes, indicating how during quiet traffic the two remaining chambers process traffic well. The remainder of the curve, however, is stretched to the right, with almost $16 \%$ of the ships experiencing a passage time of over 5 hours.

Before considering chamber downtimes for drainage, it is necessary to first assign the drainage downtimes of the removed chamber to one of the remaining, functioning, chambers. For this simulation, all drainage downtimes associated with the removed chamber were assigned to the smallest of the two remaining chambers since it is closest in size to the one removed, and therefore one may assume that the drainage downtime duration will be roughly equivalent (a large chamber could reach a higher flow rate and thus shorter drainage downtime). The results from Table 2 depict a huge increase in maximum, average and median passage times. Downtime associated with drainage appears to have an extremely negative influence on lock traffic handling 
TABLE 2. PASSAGE TIMES FOR THE DIFFERENT $2 \mathrm{C}$ SIMULATIONS, RELATIVE TO THE LOGGED PASSAGE TIMES FROM HISTORICAL DATA $(100 \%)$.

\begin{tabular}{l|cc} 
Passage time & $100 \%$ uptime & drainage downtimes \\
\hline Maximum & $188 \%$ & $509 \%$ \\
Average & $269 \%$ & $451 \%$ \\
Median & $235 \%$ & $328 \%$
\end{tabular}

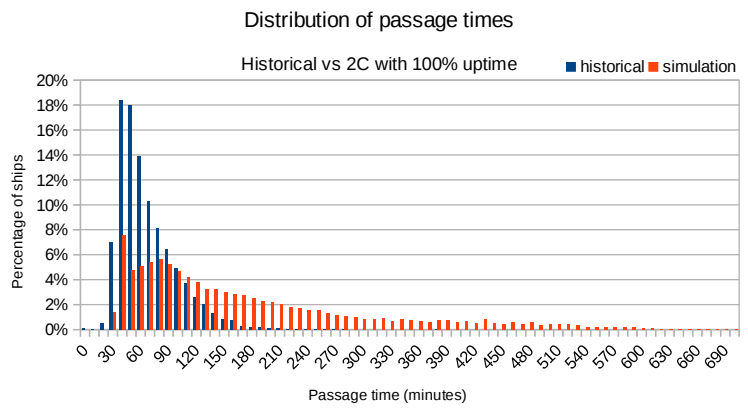

Figure 4. Distribution of passage times at the lock for both the historical data (blue) and the 2C simulation with $100 \%$ uptime (red).

efficiency. The increase of the median passage time is significantly smaller than the increase of the maximum and average passage time. This indicates how the tail of the passage time distribution is very long, but contains only a small percentage of the ships, a fact confirmed by the results illustrated within Figure 6.

\section{Optimization}

MOGLi contains an optimization library that enables significant reductions of passage times by changing the order in which ships are transferred and the chambers within which they are processed. Due to the case study's size with a time horizon of three months and a total of almost 16,000 ship passages, exact solvers prove an inviable approach. Therefore, the meta-heuristic library was employed to improve the results obtained by the simulation. All results were obtained with a Variable Neighbourhood Search evaluating 10 neighbourhoods under a dynamic weighted objective function. The time limit for the optimization was set to 24 hours. The optimization results for the $2 \mathrm{C}$ setup are reported below.

When assuming a dedicated drainage system (100\% uptime) for the $2 \mathrm{C}$ setup, passage times approximately doubled compared to the historical data. Table 3 presents the effect of applying optimization to this solution. When comparing to the historical data, the average passage time and median still double, whereas the maximum passage time increases by $40 \%$ (rather than $88 \%$ for the simulation). Compared to the simulation, optimizing traffic handling reduces the maximum passage time by approximately $25 \%$, while the average passage time and median are reduced by $17 \%$ and $8 \%$ respectively. Figure 5 illustrates the change in passage time distribution between the simulation and optimization
TABLE 3. PASSAGE TIMES AFTER OPTIMIZATION COMPARED TO LOGGED HISTORICAL DATA AND SIMULATION RESULTS.

\begin{tabular}{l|cc|cc} 
& \multicolumn{2}{|c|}{ vs Historical } & \multicolumn{2}{c}{ vs Simulation } \\
Passage time & $100 \%$ uptime & spuien & $100 \%$ uptime & spuien \\
\hline Maximum & $140 \%$ & $370 \%$ & $75 \%$ & $73 \%$ \\
Average & $224 \%$ & $390 \%$ & $83 \%$ & $87 \%$ \\
Median & $217 \%$ & $307 \%$ & $92 \%$ & $94 \%$
\end{tabular}

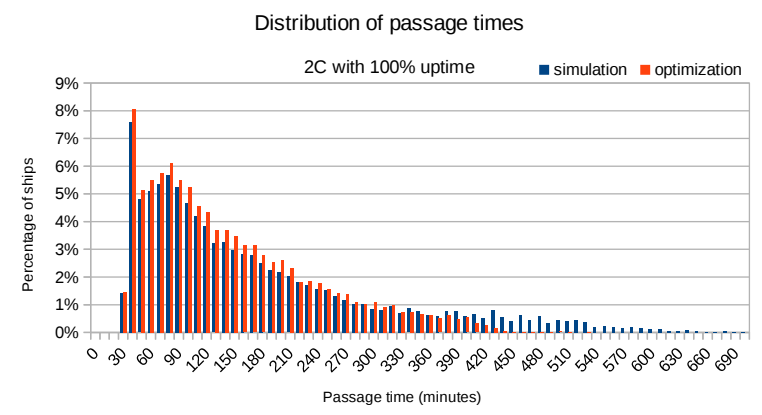

Figure 5. Distribution of passage times at the lock for both the simulation (blue) and optimization (red) the 2C setup with $100 \%$ uptime.

results for this case. The optimization's distribution tail is significantly shorter and the passage times have clearly shifted towards the beginning of the curve, confirming the aggregated results from Table 3 .

When drainage downtimes are included, the optimization obtains similar relative reductions of passage times. Maximum, average and median passage times are reduced by $27 \%, 13 \%$ and $6 \%$ respectively compared to the simulation. In absolute numbers, however, the decrease of the maximum passage time is a massive 8 hours and 30 minutes. The significant reduction of the passage time distribution's tail after optimization is also evident from Figure 6.

\section{Conclusion}

MOGLi, a simulation and optimization tool for ship lock scheduling was presented. It was applied to a case study where the smallest chamber of a three-chamber ship lock will be destroyed and replaced by a new, larger chamber. After conducting careful data analysis on historical data containing both low and peak traffic periods, the parameters for MOGLi were determined and validated by simulating the current operations at the lock. The simulation proved to be accurate both for maximum, average and median passage times, and for the overall passage time distribution. The influence of removing the smallest chamber on traffic handling at the lock was simulated. The simulation exhibited a $200-500 \%$ increase in maximum passage times at the lock, depending on whether or not a dedicated drainage system is present to reduce the water level at the upstream side of the lock during periods of heavy rainfall. This could be reduced to a $40-400 \%$ increase by introducing advanced optimization techniques to improve traffic handling at the lock. 


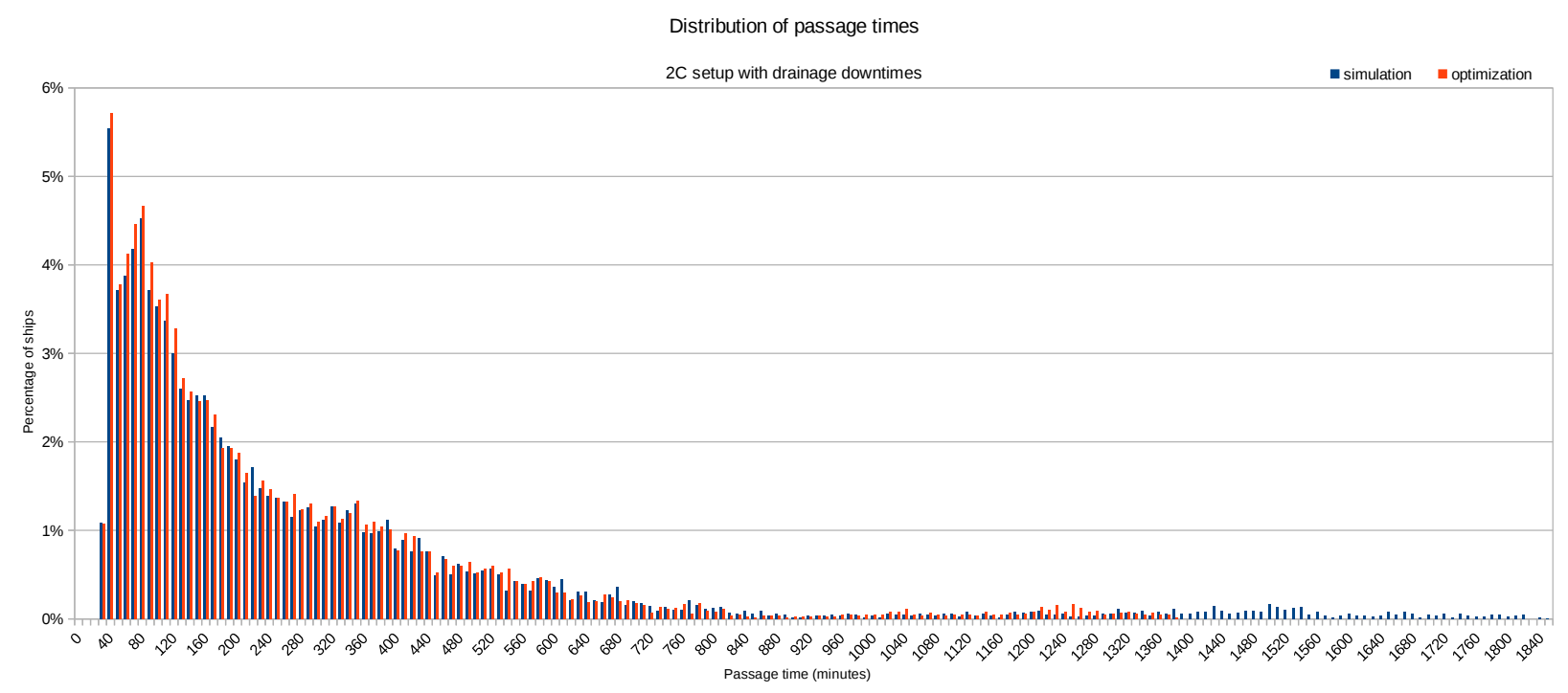

Figure 6. Distribution of passage times at the lock for both the simulation (blue) and optimization (red) of the 2C setup, including drainage downtimes.

The obtained results indicate that there exist two promising avenues for maintaining smooth traffic handling at the lock after the destruction of the smallest chamber. Firstly, it may prove worthwhile to investigate the required additional investments associated with constructing a dedicated drainage system for the locks, so that drainage downtimes no longer exist. Secondly, it could prove interesting to apply a thorough optimization of traffic handling at the locks to significantly reduce passage times rather than maintaining pre-existing policies. To ensure maximum quality of service for the shippers passing through the lock, both may be combined.

Future research avenues include extending the models to serial and multi-step locks, investigating the performance of the algorithms and adapting them, if necessary, to the new context, interfacing the system with other software employed at locks to enable real time lock scheduling optimization.

\section{Acknowledgments}

Editorial consultation provided by Luke Connolly (KU Leuven).

\section{References}

[1] European Commission, "Communication from the commission: A sustainable future for transport - towards an integrated, technology-led and user friendly systems." 2009. [Online]. Available: http://ec.europa.eu/transport/media/publications/doc/2009_future_of_ transport_en.pdf

[2] European Commission, "Transport white paper: roadmap to a single european transport area towards a competitive and resource efficient transport system." 2011. [Online]. Available: http://eurlex.europa.eu/LexUriServ/LexUriServ.do?uri=COM:2011:0144:FIN: EN:PDF
[3] A. Caris, S. Limbourg, C. Macharis, T. van Lier, and M. Cools, "Integration of inland waterway transport in the intermodal supply chain: a taxonomy of research challenges," Journal of Transport Geography, vol. 41, pp. 126-136, Dec. 2014.

[4] J. Verstichel, P. De Causmaecker, F. C. Spieksma, and G. Vanden Berghe, "Exact and heuristic methods for placing ships in locks," European Journal of Operational Research, vol. 235, no. 2, pp. 387 398, 2014.

[5] J. Verstichel, P. De Causmaecker, F. C. Spieksma, and G. Vanden Berghe, "The generalized lock scheduling problem: An exact approach," Transportation Research Part E: Logistics and Transportation Review, vol. 65, pp. 16-34, 2014.

[6] J. Verstichel, J. Kinable, P. De Causmaecker, and G. Vanden Berghe, "A Combinatorial Benders decomposition for the lock scheduling problem," Computers \& Operations Research, vol. 54, pp. 117-128, 2015.

[7] Gurobi Optimization, Inc., "Gurobi optimizer reference manual," 2015. [Online]. Available: http://www.gurobi.com

[8] "IBM ILOG CPLEX Optimizer." [Online]. Available: http://www01.ibm.com/software/integration/optimization/cplex-optimizer/

[9] N. Mladenović and P. Hansen, "Variable neighborhood search," Computers \& Operations Research, vol. 24, no. 11, pp. 1097-1100, 1997.

[10] S. Kirkpatrick, C. D. Gelatt, and M. P. Vecchi, "Optimization by simulated annealing," Science, vol. 220, no. 4598, pp. 671-680, 1983.

[11] E. K. Burke and Y. Bykov, "The late acceptance hill-climbing heuristic," University of Stirling, Tech. Rep, 2012.

[12] M. Sniedovich and S. Voß, "The corridor method: a dynamic programming inspired metaheuristic," Control and Cybernetics, vol. 35, 2006.

[13] W. Uchacz, "Optimum time scheduling of lock traffic," Methods and Models in Automation and Robotics (MMAR), 18th International Conference on, Miedzyzdroje, 2013, pp. 393-398, 2013.

[14] T. Wauters, W. Vancroonenburg, and G. Vanden Berghe, "A guideand-observe hyper-heuristic approach to the eternity II puzzle," Journal of Mathematical Modelling and Algorithms, vol. 11, no. 3, pp. 217-233, 2012. 